\title{
Where Does Energy Go in Electron Energy Loss Spectroscopy of Nanostructures?
}

Gabriel D. Bernasconi, ${ }^{\dagger, \#}$ Jérémy Butet, ${ }^{*} \dagger, \# \odot$ Valentin Flauraud, ${ }^{\ddagger}$ Duncan Alexander, ${ }^{\S}$ Juergen Brugger, ${ }^{\dagger}$ and Olivier J. F. Martin ${ }^{\dagger}$

Nanophotonics and Metrology Laboratory, ${ }^{\dagger}$ Microsystems Laboratory, and ${ }^{\S}$ Interdisciplinary Center for Electron Microscopy, Swiss Federal Institute of Technology Lausanne (EPFL), 1015 Lausanne, Switzerland

Supporting Information

ABSTRACT: Electron energy loss spectroscopy is a method of choice for the characterization of both the spatial and spectral properties of localized surface plasmon resonances. The energy lost by the impinging electrons is commonly explained by the Lorentz force acting on their motion. Here, we adopt another point of view to compute the electron energy loss spectra. Coupling the energy conservation law with full-wave electromagnetic computations based on a surface integral equation method, we derive the electron energy loss spectra directly from two dissipative processes, namely, absorption and scattering. This antenna-based approach is applied to nanostructures with different sizes and materials, showing an excellent agreement with experimental observation

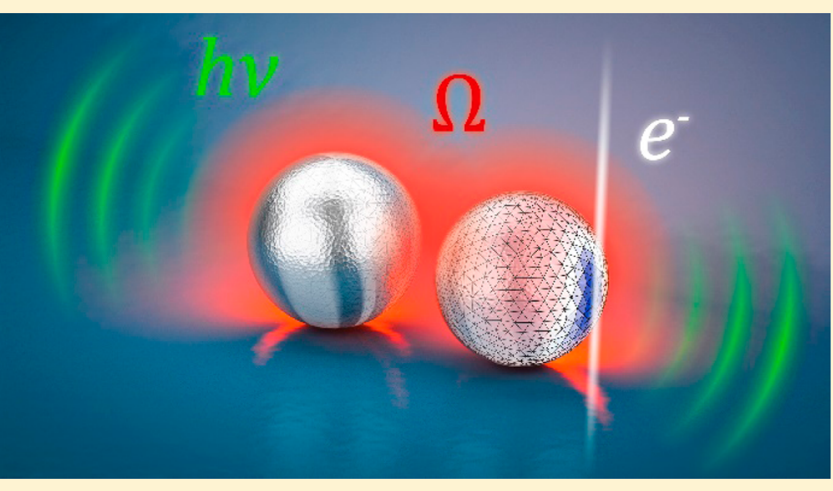
and computations based on the evaluation of the Lorentz force. This formalism permits the easy separation of absorption losses in the nanostructures forming a coupled system and reveals the subtle interplay between absorption and scattering, which are controlled by the materials, the nanostructure size, and the energy range.

KEYWORDS: electron energy loss spectroscopy, gold, silver, nanoparticles, surface integral equation method, transmission electron microscopy

$\mathrm{E}$ lectron energy loss spectroscopy (EELS) is a wellestablished experimental technique based on the measurement of the energy lost by an electron owing to its interaction with a sample. ${ }^{1,2}$ The implementation of EELS obviously requires the use of an electron beam and is in general performed in a scanning transmission electronic microscope (STEM) combined with an electron spectrometer. ${ }^{1,2}$ EELS is an important experimental technique for both solid and surface sciences, combining very high spatial and energy resolutions. ${ }^{3-7}$ Indeed, the spatial resolution of EELS is mainly related to the electron beam size, which can be squeezed to subangstrom dimensions, allowing for the direct observation of single atoms. $^{3-7}$ At the same time, a high energy resolution is mandatory for the identification of various chemical species and atomic bonds present in the sample under study. ${ }^{3-7}$ In particular, the energy resolution has been increased to $\sim 10$ $\mathrm{meV}$, allowing performing vibrational spectroscopy in STEM. ${ }^{8}$ Examples of the successful utilization of EELS include the investigation of important biological molecules such as nucleic acid bases, ${ }^{9}$ confined fluids within individual nanobubbles, ${ }^{10}$ oxidation states in transition metal oxides, ${ }^{11}$ single-atom dopants in graphene, ${ }^{12}$ and light elements (alkali metal and halogen). ${ }^{13}$

The characterization of localized surface plasmon resonances (LSPRs) using swift electrons in STEM has also become an important research topic during the past decade. ${ }^{14,15}$ This is mainly due to the combination of high spatial and spectral resolutions, which enables direct mapping of the electromagnetic hot-spots associated with LSPRs. ${ }^{16}$ The interaction between the impinging electrons and the LSPR is observed either as energy lost by the electron beam, corresponding to EELS, ${ }^{16-37}$ or as cathodoluminescence (CL), ${ }^{37-41}$ which corresponds to the photon emission resulting from the radiative decay of the LSPR. EELS and CL have been widely investigated in a broad variety of plasmonic systems, including nanospheres, ${ }^{17,18}$ nanorods, ${ }^{19,20}$ nanowires, ${ }^{21,22}$ nanoprisms, ${ }^{16,23}$ nanodisks, ${ }^{24}$ nanocubes, ${ }^{25,26}$ nanosquares, ${ }^{27}$ nanodecahedra, ${ }^{28}$ nanostars, ${ }^{29}$ bipyramids, $^{30}$ split-ring resonators, ${ }^{31}$ nanodimers, ${ }^{32-34}$ trimers, ${ }^{35}$ chains of nanoparticles, ${ }^{36}$ and nanodolmens. $^{37}$ Recently, EELS was used to control the precise placement of gold nanorods in coupled systems obtained by capillary assembly. ${ }^{42}$ Furthermore, EELS has been used to probe LSPR kinetics and damping in single plasmonic nanostructures. ${ }^{29}$ Indeed, the LSPR quality factor and dephasing time depend upon the total losses, including radiative and nonradiative channels. ${ }^{43}$ In order to support these experimental observations, numerical methods for

Received: October 4, 2016

Published: December 5, 2016 
nanophotonics have been extended to describe the interaction between plasmonic systems and propagating electrons. ${ }^{14}$ For example, the discrete dipole approximation, ${ }^{4-46}$ the $3 \mathrm{D}$ Green's dyadic method, ${ }^{47}$ the finite difference time domain method, ${ }^{48}$ the discontinuous Galerkin time-domain method, ${ }^{49}$ the boundary element methods $(\mathrm{BEM})^{50-52}$ - in particular the MNPBEM Matlab toolbox ${ }^{53}$-and the finite element method ${ }^{32}$ have been used for computing EELS and CL spectra.

In order to obtain more insights into the corresponding interaction mechanisms, the electron energy loss (EEL) spectra and maps have been related to a well-established physical quantity, the local density of states (LDOS).$^{54-57}$ It was shown that EELS is sensitive to the component of the LDOS parallel to the electron propagation direction and that the EEL spectra are related to the total LDOS, while CL is related to the radiative part only. ${ }^{54-57}$ Different tomography methods have been proposed for the complete reconstruction of the plasmonic eigenmodes, ${ }^{58,59}$ and a circuit model has been applied to understand the evolution of plasmon resonances observed with EELS. ${ }^{60,61}$ However, until now, all the numerical methods developed for the computation of EEL spectra are based on the direct computation of the work done by the Lorentz force on the electron. This corresponds to a description from the point of view of the electron, thus somehow hiding the role played by the intrinsic properties of the plasmonic nanostructure in the EEL spectrum. It should be noted that the methods based on a modal decomposition also use the evaluation of the Lorentz force acting on the electron as a starting point. ${ }^{54-57}$ In parallel, the relation between EELS and absorption and scattering cross sections has been investigated. It was shown that, in specific cases, the EEL probability can be equal to only the radiative loss probability, i.e., to CL, such as the case of $\mathrm{Al}_{2} \mathrm{O}_{3}$ nanoparticles. ${ }^{62}$ Further, it was formally proved that EELS is related to the extinction (the sum of absorption and scattering) cross sections in the case of small nanospheres. ${ }^{14}$ Slight shifts observed between EELS and CL spectra have been explained by the differences in the scattering and absorption cross sections. ${ }^{37,40,57}$ However, a systematic investigation of the relationship between EEL spectra and the absorption/scattering properties of the nanostructures has not yet been done, especially in the retarded case and for coupled nanostructures, although important advances have recently been made in this direction. ${ }^{40}$

In this article, we evaluate the EEL spectrum directly from the properties of the plasmonic nanostructure. On the basis of energy conservation, the EEL spectrum is related to the fundamental electromagnetic properties of the plasmonic nanostructure-which can be viewed as an optical antenna ${ }^{63}$-namely, absorption and scattering. Here, the antenna theory is implemented in a surface integral equation (SIE) method, ${ }^{64}$ but this can be easily adapted for other numerical techniques. Spherical nanoparticles are considered first in order to introduce the antenna theory in a simple case. The roles of both the nanoparticle size and the constituting metal are then discussed in detail. Simple coupled plasmonic systems, i.e., nanodimers, are also investigated using the antenna theory, demonstrating that the contribution of each nanoparticle in the total EEL spectrum can be clearly identified in this framework. Finally, silver nanodiscs have been fabricated, and their EEL spectra have been interpreted using the novel theoretical approach.

\section{RESULTS}

Theory. A swift electron propagating in a homogeneous medium generates an electromagnetic field that can probe matter with a high spatial resolution. Contrary to a planewave, which is monochromatic but spatially extended, the electromagnetic field associated with a propagating electron is strongly localized close to its trajectory but is associated with a broad spectrum. The spectral components of the electric field $\mathbf{E}(\mathbf{r}, \omega)$ and magnetic field $\mathbf{H}(\mathbf{r}, \omega)$ associated with an electron propagating along the $z$-axis are conveniently given by analytical expressions involving modified Bessel functions: ${ }^{65}$

$$
\begin{aligned}
& \mathbf{E}(\mathbf{r}, \omega)=\frac{2 q \omega \mathrm{e}^{i \omega z / v}}{v^{2} \gamma_{\varepsilon} \varepsilon}\left[\frac{i}{\gamma_{\varepsilon}} K_{0}\left(\frac{\omega R}{v \gamma_{\varepsilon}}\right) \hat{z}-K_{1}\left(\frac{\omega R}{v \gamma_{\varepsilon}}\right) \hat{R}\right] \\
& \mathbf{H}(\mathbf{r}, \omega)=-\frac{2 q \omega \mathrm{e}^{i \omega z / v}}{v c \gamma_{\varepsilon}} K_{1}\left(\frac{\omega R}{v \gamma_{\varepsilon}}\right) \hat{\varphi}
\end{aligned}
$$

where $\gamma_{\varepsilon}=1 / \sqrt{1-\varepsilon v^{2} / c^{2}}$ is the Lorentz contraction factor, $v$ is the electron velocity, $\varepsilon$ is the relative permittivity of the medium, $q$ is the electron charge, $K_{0}$ and $K_{1}$ are the modified Bessel functions of the second kind, $\hat{\mathbf{R}}$ is the radial unit vector, $R$ and $z$ are respectively the perpendicular distance to and along the electron trajectory $(\mathbf{r}=(R, z))$, and $\hat{\varphi}$ is the azimuthal unit vector. These formulas are directly used in all the numerical methods implemented in the frequency domain for the evaluation of EEL spectra. In this work, we use a SIE method previously applied to the computation of the interaction of nanostructures with incoming planewaves and radiative dipoles. $^{66}$ This method has been proven to be very accurate for the description of the electrodynamic response of plasmonic systems, even under resonant conditions. ${ }^{64,66}$ The nanostructure's surface is discretized with triangular mesh elements, and using surface integral equations, the electric and magnetic fields in the entire space (inside and outside the nanoparticle) are related to fictitious electric and magnetic surface currents. The incident conditions are defined using eqs 1 and 2 , and the induced field, resulting from the interaction between the electromagnetic field generated by the electron and a scatterer, can be accurately determined. The spectral loss probability, the quantity measured in standard EELS experiments, is generally expressed invoking the Lorentz force exerted by the induced electric field $\mathbf{E}^{\text {ind }}(\mathbf{r}, \omega)$ on the incident electron. Considering a straight line trajectory $\mathbf{r}_{\mathrm{e}}(t)$ and a constant electron velocity $\mathbf{v}$ (the so-called nonrecoil approximation ${ }^{13}$ ), the energy loss is expressed as ${ }^{67}$

$$
\Delta E=e \int \mathrm{d} t \mathbf{v} \cdot \mathbf{E}^{\text {ind }}(\mathbf{r}, t)=\int_{0}^{\infty} \hbar \omega \Gamma(\omega) \mathrm{d} \omega
$$

where

$$
\left.\Gamma(\omega)=\frac{e}{\pi \hbar \omega v} \int \operatorname{Re}\left\{\left(\mathbf{v e} e^{-i \omega t}\right) \cdot \mathbf{E}^{\text {ind }}(\mathbf{r}, \omega)\right)\right\} \mathrm{d} l
$$

is the loss probability given per unit of frequency $\omega$. A priori, EEL spectra can be computed with any numerical method suitable for the evaluation of the induced electric field. ${ }^{68}$ All these numerical methods are based on the evaluation of the work done by the Lorentz force against the propagation of the electron. The current implementation of the SIE method for the evaluation of the EEL spectra has been tested and compared to the BEM (with the MNPBEM package); $52-54$ 

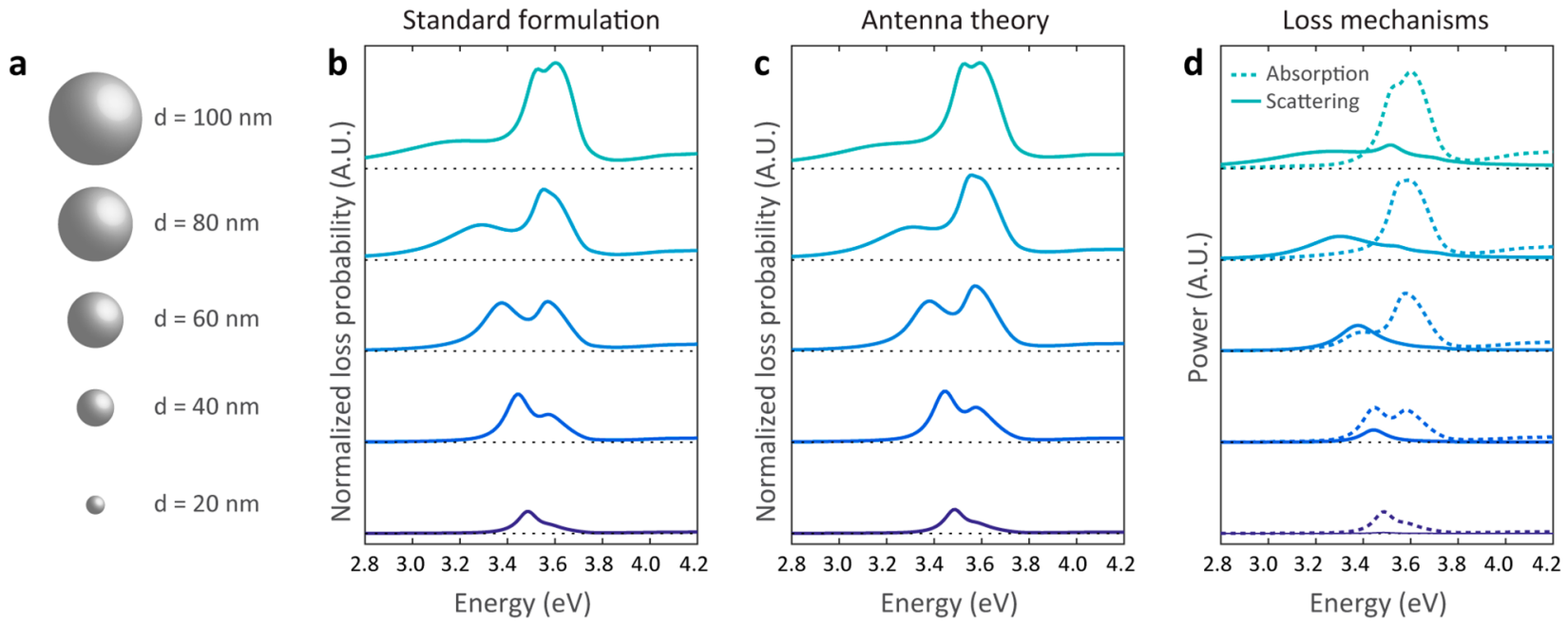

Figure 1. (a) EEL spectra for silver nanospheres in vacuum. (b) Normalized loss probability evaluated using the standard formulation corresponding to eq 4. (c) Normalized loss probability evaluated using the antenna theory corresponding to eq 5. (d) Absorbed power (dashed lines) and scattered power (solid lines) shown as functions of the energy. The minimal distance between the nanoparticle surface and the electron trajectory is fixed to 4 $\mathrm{nm}$. The incident electron energy is $300 \mathrm{keV}$.

see Figure S1 in the Supporting Information. In the following, the method based on the evaluation of the Lorentz force is referred to as the standard formulation and the corresponding loss probability is denoted as $\Gamma_{\text {stand }}(\omega)$.

In this article, we introduce another point of view to describe EELS by combining antenna theory with energy conservation. Indeed, an electron does not lose energy as it propagates in a homogeneous medium with a velocity lower than the speed of light in this medium, i.e., without Cherenkov radiation. ${ }^{14}$ As a consequence, the unique possibility for the electron to lose energy is through its interaction with the plasmonic nanostructures close to its trajectory. The energy transferred from the electron to the nanostructure can then be dissipated either via heat generation in the nanostructure or by radiation into the far field. Note that a small amount of the energy absorbed in the nanostructure can be tied to the generation of hot excitons, which may leave the plasmon nanostructures without generating any phonons. ${ }^{69,70}$ This effect is not considered in the present work. The latter dissipative process corresponds to the emission of photons and is known as CL. ${ }^{38-41}$ Due to energy conservation, the power lost by a beam of temporally well-separated electrons, i.e., if the time separating two impinging electrons is much longer than the dissipation of the energy generated in the nanostructure by one electron, $P_{\text {loss }}(\omega)$ can be written as

$$
P_{\text {loss }}=P_{\mathrm{sca}}+P_{\mathrm{abs}}
$$

where $P_{\text {sca }}$ is the power scattered by the nanostructure and $P_{\text {abs }}$ is the absorbed power, corresponding to the ohmic losses. The absorbed power $P_{\text {abs }}$ is easily computed as ${ }^{71}$

$$
P_{\mathrm{abs}}(\omega)=\frac{1}{2} \int_{\Omega} \mathrm{d} V \operatorname{Re}\{\sigma\}\left|\mathbf{E}^{\mathrm{ind}}(\mathbf{r}, \omega)\right|^{2}
$$

where the integration is performed over the volume $\Omega$ of the plasmonic system. The conductivity $\sigma$ is expressed as ${ }^{71}$

$$
\sigma(\omega)=-i \omega \varepsilon_{0}\left(\varepsilon_{\mathrm{m}}-1\right)
$$

where $\varepsilon_{\mathrm{m}}$ is the complex dielectric constant of the metal and $\varepsilon_{0}$ is the vacuum permittivity. The dielectric constants of gold and silver are extrapolated from experimental results in the present work. ${ }^{72}$ The scattered power $P_{\text {sca }}$ is evaluated by integrating the Poynting vector $\mathbf{S}(\mathbf{r}, \omega)$ over a surface $A$ enclosing the nanostructure,

$$
P_{\mathrm{sca}}(\omega)=\int_{A} \mathrm{~d} \mathbf{A} \cdot \mathbf{S}(\mathbf{r}, \omega)
$$

Note that scattering denotes here the electromagnetic radiation, not the electron scattering by the LSPR. In this framework, the loss probability $\Gamma_{\text {loss }}(\omega)$ per unit of frequency $\omega$ is proportional to $\frac{N}{\hbar \omega^{2}} P_{\text {loss }}(\omega)$, where $N$ is the constant number of incident electrons per time unit. The loss probability $\Gamma_{\text {loss }}(\omega)$ can then be related to two dissipation processes, namely, scattering and absorption. Furthermore, in the case of coupled plasmonic systems, the absorption in each individual nanoparticle can be computed separately by an adequate definition of the integration volume as shown below.

Before discussing several examples in detail, it is worth saying that the antenna point of view proposed here is compatible with the common description of EELS based on the LDOS. $^{54-57}$ Indeed, it was recently demonstrated that the CL spectra are related to the radiative LDOS, while EEL spectra are related to the full LDOS, including the radiative and the nonradiative components. ${ }^{56}$ However, there was a long controversy about the link between the LDOS and EELS and how they are intimately related to each other. ${ }^{54-57}$ It is now well-established that EELS is able to probe only the component of the LDOS parallel to the electron trajectory. ${ }^{54-57}$ This is generally attributed to the scalar product between the electron velocity and induced electric field in the integrand of eq 4 . This scalar product is also invoked to explain that EELS is blind to some of the electromagnetic hot-spots, as the ones observed in the gap of dipolar nanoantennas, due to an inappropriate orientation of the electric field. ${ }^{54-57,73}$ This has been intensively discussed in the frame of the modal decomposition of the EEL spectra. ${ }^{56}$ While this point of view is correct, the approach proposed in this article emphasizes that the inability of EELS to reveal some of the hot-spots is indeed due to the specific symmetry of the incident field associated with the incoming electrons. 

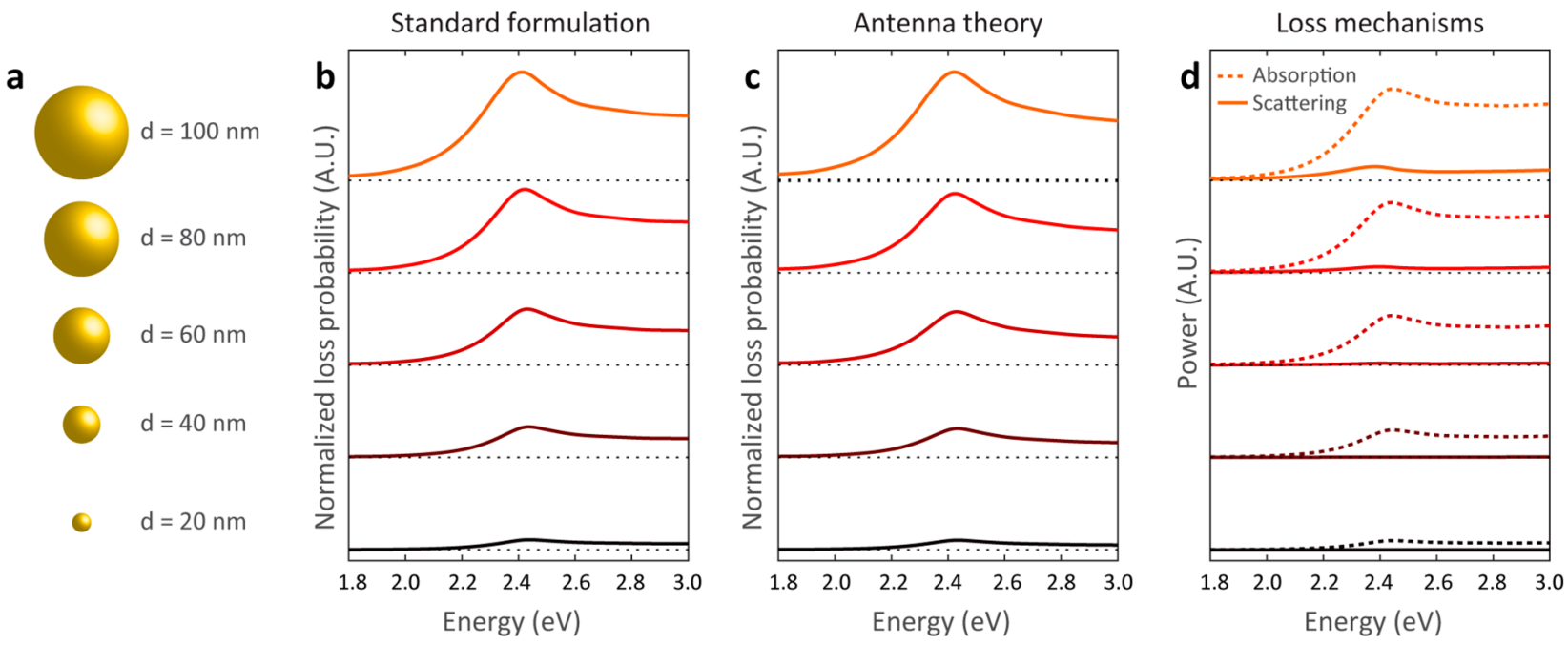

Figure 2. (a) EEL spectra of gold nanospheres in vacuum. (b) Normalized loss probability evaluated using the standard formulation corresponding to eq 4. (c) Normalized loss probability evaluated using the antenna theory corresponding to eq 5. (d) Absorbed power (dashed lines) and scattered power (solid lines) shown as functions of the energy. The minimal distance between the nanoparticle surface and the electron trajectory is fixed to 4 $\mathrm{nm}$. The incident electron energy is $300 \mathrm{keV}$.

Silver Nanoparticles. We start with simple plasmonic systems, namely, silver nanospheres in vacuum. The sphere diameters range from $d=20 \mathrm{~nm}$ to $d=100 \mathrm{~nm}$, Figure 1a. The incident electron energy is fixed to $300 \mathrm{keV}$. The impact parameter is $b=d+4 \mathrm{~nm}$, meaning that the minimal distance between the sphere surface and the electron trajectory is $4 \mathrm{~nm}$. The corresponding EEL spectra have been computed using the standard formulation, i.e., the evaluation of the work done on the electron by the induced field, and the antenna point of view proposed in this article. A very good agreement between the two formulations is observed, confirming the validity of the antenna point of view for EELS. For the $20 \mathrm{~nm}$ silver nanoparticle, the EEL spectra are composed of one maximum close to $3.49 \mathrm{eV}$ with a shoulder at higher energy. The Mie theory reveals that the mode peaking at $3.49 \mathrm{eV}$ corresponds to the electric dipole mode and the shoulder is due to the contribution of higher modes, mainly the electric quadrupole mode. $^{74}$ As the nanoparticle size increases, the dipolar mode red-shifts and its contribution to the EEL spectra evolves; see Figure 1. The contribution of the electric dipole mode to the EEL spectra is maximal for a sphere diameter $d$ between 40 and $60 \mathrm{~nm}$. As the nanoparticle size increases, the contribution of the electric quadrupole mode becomes more significant and a new (electric octupole) mode appears for the largest nanoparticles at an energy close to that of the quadrupolar mode, explaining the peak at $3.6 \mathrm{eV}$ for $d=100 \mathrm{~nm}$.

Having briefly described the evolution of the EEL spectra with the size of silver nanoparticles, we now turn our attention to the cornerstone of the approach proposed in this article: the decomposition of the EEL spectra into the different energy loss channels. The ohmic losses (dashed lines) and the scattered power (full lines) are shown in Figure 1d. First of all, it is worth noticing that the maxima are slightly shifted relatively to the ones observed in the EEL spectra. Indeed, the EEL spectra correspond to the probability that an electron undergoes an energy loss equal to $\hbar \omega$. In other words, the EEL spectra reveal the number of loss events. On the other hand, the total dissipated power is the convolution between the loss probability and the energy loss during each event, proportional to $\hbar \omega$, explaining the small shift between the two kinds of spectra. This shift is even higher for $20 \mathrm{~nm}$ aluminum nanoparticles, which are resonant in the $\mathrm{UV}$ close to $10 \mathrm{eV}$; see Figure S2 in the Supporting Information. At this energy, an energy shift as high as $0.5 \mathrm{eV}$ is observed between the maximum of the EEL spectrum and the maximum of the total energy loss. For the smallest silver nanoparticles, the energy dissipation is dominated by the internal absorption, in agreement with previous discussions. ${ }^{17}$ However, the scattering also plays a more important role as the nanoparticle increases. For example, the peak at $3.38 \mathrm{eV}$ observed in the EEL spectrum of a $60 \mathrm{~nm}$ silver nanoparticle is mainly due to scattering, and not to absorption. This peak is associated with the electric dipole mode. In the case of $100 \mathrm{~nm}$ silver nanoparticle, the electric dipole mode is shifted to lower energy and the scattering associated with this mode is the main energy dissipation process for energy lower than $3.4 \mathrm{eV}$. At the same time, new modes, which are strongly absorbing, appear close to $3.6 \mathrm{eV}$, resulting in EEL spectra also dominated by absorption. Note that the small scattering peak observed at $3.5 \mathrm{eV}$ corresponds to the electric quadrupole mode.

Gold Nanoparticles. As expressed by eqs 6 and 7, the ohmic losses are proportional to the imaginary part of the dielectric constant of the metal. Therefore, the metal constituting the investigated nanostructures is an important parameter in EELS, beyond its influence on the resonant frequencies of the observed modes. Furthermore, the penetration of the electromagnetic field inside the nanoparticle also depends on the dielectric constant, because this physical quantity is involved in the boundary conditions describing the electromagnetic field behavior at the nanoparticle surface. To investigate the influence of the metal dielectric constant on EELS measurements, EEL spectra of gold nanoparticles with diameter $d$ ranging from 20 to $100 \mathrm{~nm}$ have been evaluated in vacuum using the standard formulation and the antenna theory; see Figure 2. All the EEL spectra are characterized by two distinct features. First, a maximum at $2.41 \mathrm{eV}$, associated with the excitation of LSPRs and with an increase of the internal electric field, is observed. The second feature is a plateau for energy higher than $3 \mathrm{eV}$, corresponding to the excitation of electronic interband transitions from the d-band to the 

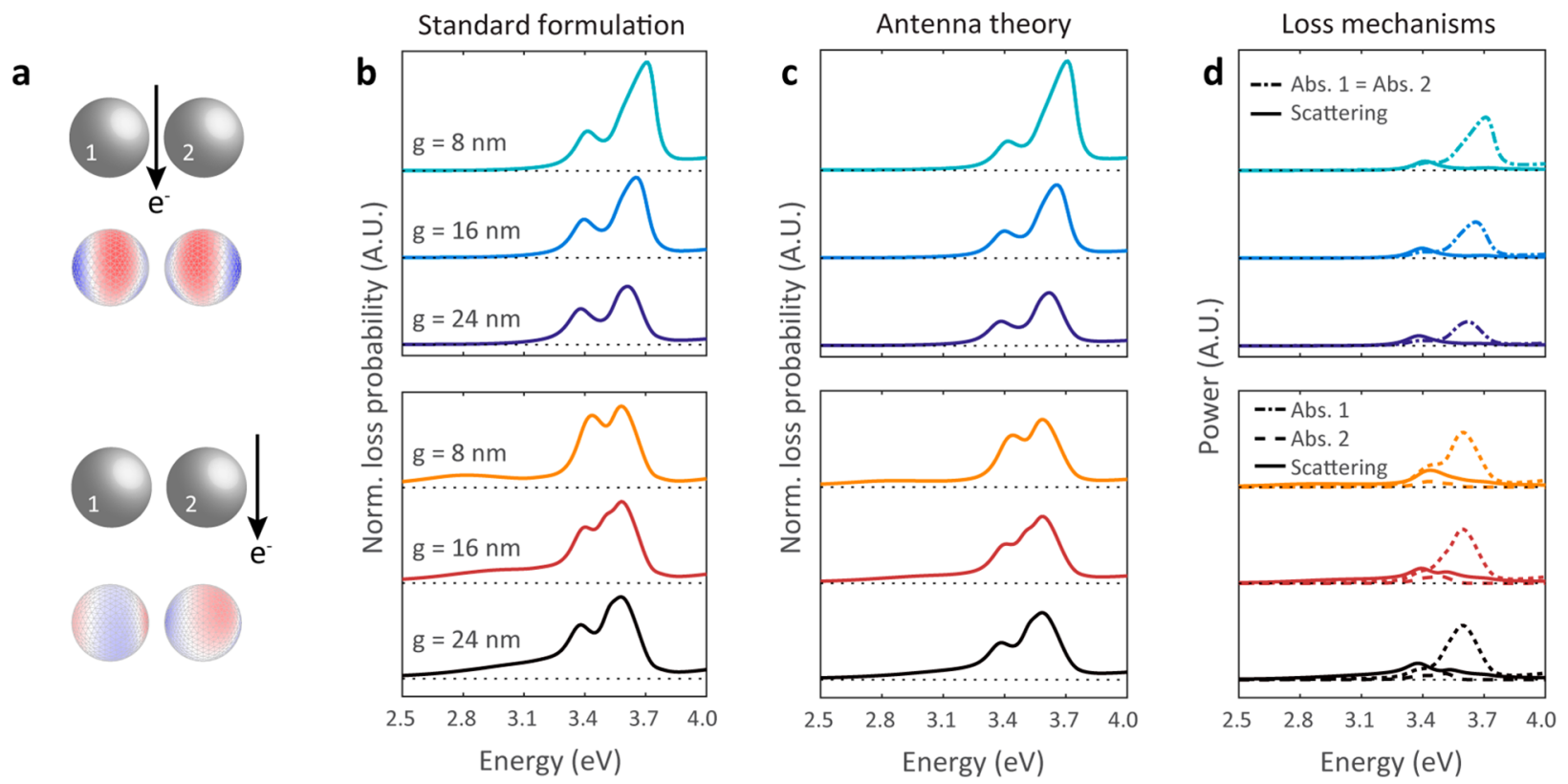

Figure 3. (a) EELS of silver nanodimers in vacuum for an electron trajectory passing through the dimer center and perpendicular to the dimer main axis (top panels) and for an electron trajectory passing close to the dimer extremity (the minimal distance between the nanoparticle surface and the electron trajectory is fixed to $4 \mathrm{~nm}$ in this case) and perpendicular to the dimer main axis (bottom panels). (b) Normalized loss probability evaluated using the standard formulation corresponding to eq 4. (c) Normalized loss probability evaluated using the antenna theory corresponding to eq 5. (d) Absorbed power in nanoparticle 1 (dash-dotted lines) and in nanoparticle 2 (dashed lines) and the scattered power (solid lines) shown as functions of the energy. The incident electron energy is $300 \mathrm{keV}$.

hybridized sp-band and to an increase of the imaginary part of the gold dielectric constant. The nanoparticle size modifies the relative weight of these two features in the EEL spectra. The antenna theory reveals that, for all the studied nanoparticle diameters, the energy is mainly converted into heat by ohmic losses (see Figure 2d) and that the scattering is weak, resulting in a limited CL signal. Indeed, scattering corresponds approximately to $15 \%$ of the energy lost at $2.41 \mathrm{eV}$ by a 100 $\mathrm{nm}$ gold nanoparticle, the other part being lost by heat dissipation.

Silver Dimers. In the EELS of the isolated nanoparticles, the energy is dissipated either by scattering or by absorption. The case of coupled nanostructures is a bit more complex. Indeed, the absorption is decomposed into the ohmic losses occurring in each nanoparticle constituting the coupled plasmonic system. The absorption in each nanoparticle can be easily computed considering the appropriate volume for the integration occurring in eq 6 . The simplest coupled plasmonic system is composed of two spherical nanoparticles separated by a short distance. The EEL spectra of silver dimers made of two $80 \mathrm{~nm}$ silver nanospheres with gaps $g=8,16$, and $24 \mathrm{~nm}$ have been evaluated using the standard formalism and the antenna theory (Figure 3). When the incident electron trajectory passes through the dimer center and is perpendicular to the dimer main axis (top panels), the EEL spectra are composed of two peaks, which red-shift as the gap between the two nanoparticles increases. This behavior is explained by the antibonding nature of the modes, where surface polarization charges with the same signs stand at each side of the gap (see the charge distributions in Figure 3a), excited in this configuration. Indeed, due to the symmetry properties of the electromagnetic field generated by the impinging electrons, the bonding modes (where surface polarization charges with opposite signs stand at each side of the gap) cannot be excited. This has been intensively discussed in the past, leading to the conclusion that the Lorentz force associated with the bonding modes vanishes since the corresponding electric field is perpendicular to the electron trajectory in this case. ${ }^{54,55} \mathrm{~A}$ priori, in this framework one cannot determine whether a given mode is not excited or is instead excited but not probed by the electron beam. In comparison, the absorbed and scattered power are not directly related to the induced local field orientation (contrary to the Lorentz force). It follows that the vanishing signal in the gap reveals that the bonding modes are simply not driven by the incident electrons and therefore do not provide additional channels for energy dissipation. The standard formalism and the antenna theory lead to the same physical results, but the latter description introduces a subtle conceptual difference in the interpretation of the EEL spectra. It is interesting to note that, despite the antibonding nature of the modes, a nonnegligible part of the energy is dissipated through scattering. Furthermore, the absorption in the two nanoparticles is always identical, as expected from simple symmetry considerations. This observation confirms the validity of the antenna theory for the description of the EEL spectra.

In order to go beyond the selective excitation of the antibonding modes, the electron beam is moved close to the dimer edges with a distance of $4 \mathrm{~nm}$ between the sphere surface and the electron trajectory. In this case, the EEL spectra reveal two main peaks as well as energy losses at lower energy (between 2.5 and $3 \mathrm{eV}$ ). This feature is attributed to the contribution of the dipolar bonding mode, which was not excited in the previous configuration; see the charge distributions in Figure 3a. Note that this mode is broader than the other ones due to its high radiative losses. The absorption in the two nanoparticles reveals their relative contributions to the EEL spectra. Interestingly, it is observed that the nanoparticle close to the electron beam dissipates more 

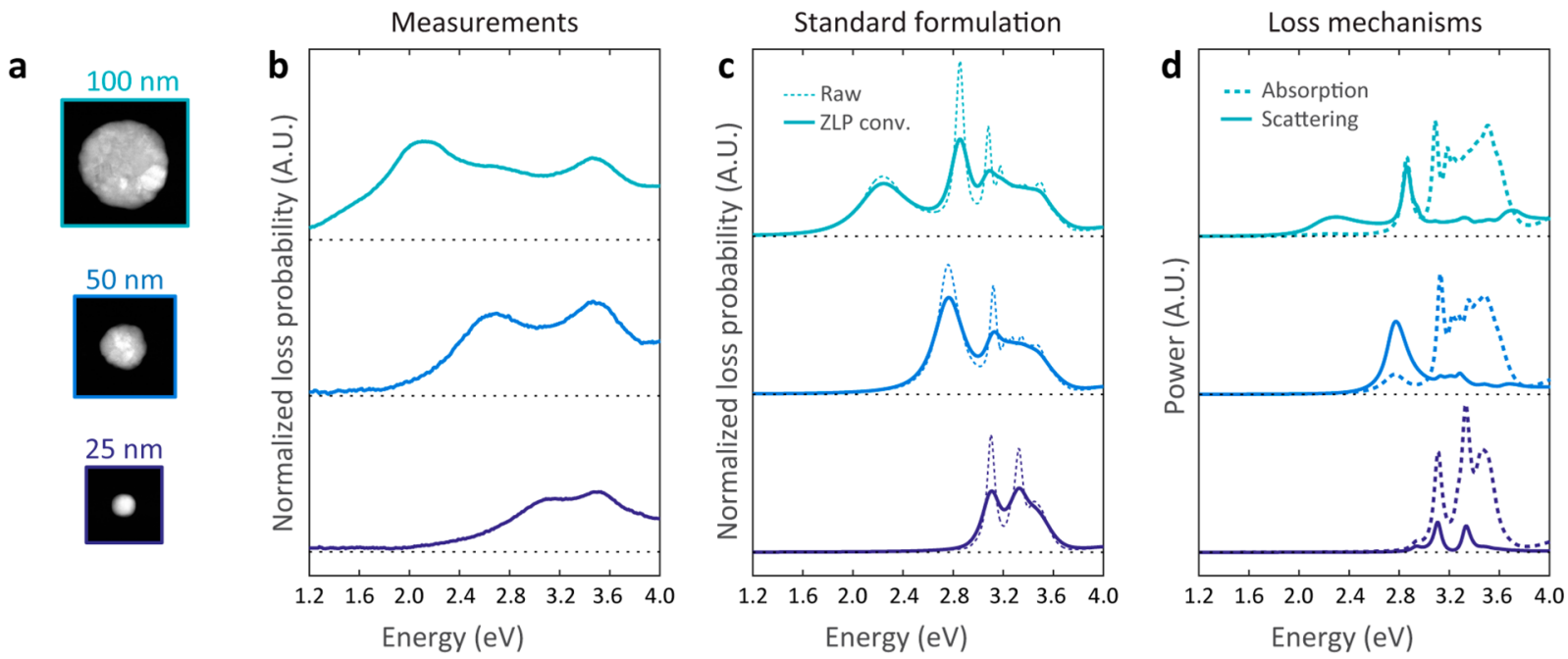

Figure 4. (a) EEL spectra of silver nanodiscs. (b) Experimental spectra. (c) Normalized loss probability evaluated using the standard formulation corresponding to eq 4 . The raw data and the convolution with the experimental zero-loss peak (ZLP) are shown in dashed lines and solid lines, respectively. (d) Absorbed power (dashed lines) and scattered power (solid lines) shown as functions of the energy. The incident electron energy is $300 \mathrm{keV}$ for both experiments and computations.

energy than the other one. This is expected since the internal electric field is higher inside this nanoparticle due to the fast spatial decay of the electric field generated by the incident electrons, eq 1 . Note that the relative contributions of the nanoparticles cannot be easily determined using the standard formalism and the evaluation of the Lorentz force, mainly because the external electric field is associated with the complete coupled system, not to only one of the nanoparticles. These results emphasize the potential of the antenna theory for the interpretation of the EEL spectra associated with coupled plasmonic systems, especially the role played by the different nanoparticles in the global response.

Comparison with Experimental Results. Finally, the numerical method proposed in this article for simulation of EELS is compared to experimental EEL spectra obtained for silver nanodiscs with various sizes, with diameter $d$ ranging from 25 to $100 \mathrm{~nm}$ and with a constant thickness of $30 \mathrm{~nm}$. As discussed previously, the energy dissipation in gold nanoparticles is mainly due to ohmic losses, and silver is chosen here to emphasize the role played by the two energy loss channels (scattering and internal absorption). The silver nanodiscs were fabricated by electron beam lithography along with a standard lift-off technique on an electron-transparent silicon nitride membrane with a thickness of $30 \mathrm{~nm}$. The EEL spectra were extracted from $30 \times 30$ pixel regions of high-resolution EELS maps allowing for an improved signal-to-noise ratio and low sample damages in comparison with a long single-point spectra acquisition. The comparison of the experimental spectra with the simulated EEL spectra convoluted with the experimental zero-loss peak (ZLP, with $0.12 \mathrm{eV}$ fwhm) shows a good agreement. The slight discrepancy is thought to come from the fact that an effective medium is used to mimic the influence of the substrate (see Methods). Especially, a gradual red-shift of the electric dipole mode as the nanodisc diameter increases and a quasi-planar mode at $3.5 \mathrm{eV}$ are observed. Furthermore, both the experimental results and the theoretical predictions reveal that the number of modes distinctly observed in the EEL spectra increases as the nanodisc size grows. Now, we turn our attention to the loss mechanisms and their evolutions with the nanodisc diameter; see Figure 4d. For the smallest studied silver nanodisc $(d=25 \mathrm{~nm})$, the energy is mainly absorbed in the nanoparticle, and the scattering remains weak over the whole spectrum. However, as the nanodisc size increases, scattering plays a more prominent role in the total energy dissipation, as observed previously in the case of the silver nanospheres. It is interesting to note that scattering mainly contributes to the EEL spectra at low energy and absorption at high energy. This observation is related to the physical properties of the modes and their coupling to far-field radiations, as emphasized by the antenna theory.

Conclusions and Perspectives. In summary, a new method for the evaluation and interpretation of the EEL spectra has been proposed. Contrary to the approaches proposed previously, the Lorentz force is not directly evaluated, but an antenna point of view is adopted instead, thus allowing distinguishing between the different loss channels provided by the nanostructure. Several examples, including gold and silver spherical nanoparticles, silver dimers with various gaps, and silver discs made by lithography, have been considered in order to confirm the validity of this approach and to demonstrate the new insights it provides. It should also be emphasized that this approach is complementary to the LDOS description proposed in several seminal articles. Indeed, the antenna theory enables determining the relative role of each nanoparticle constituting a coupled plasmonic system, while this information is hidden in the LDOS of the global system. For this reason, the antenna point of view proposed in this article will become an important theoretical tool for the subtle interpretation of EEL spectra in complex plasmonic systems, such as those supporting Fano resonances for example. ${ }^{75}$

\section{METHODS}

Simulations. A surface integral equation method is used to compute the interaction between the electromagnetic field associated with the swift electron and the plasmonic nanostructures. The nanostructure's surface is discretized with triangular mesh elements with a typical side length between 1.5 $\mathrm{nm}$ for $20 \mathrm{~nm}$ spherical nanoparticles and $8 \mathrm{~nm}$ for $100 \mathrm{~nm}$ spherical nanoparticles, and using integral equations, ${ }^{61}$ the electric and magnetic fields in the entire space (inside and 
outside the nanoparticle) are related to fictitious electric and magnetic surface currents. The same numerical method is used for the implementation of both the standard formalism and the antenna theory. For the silver nanodiscs, a homogeneous medium with an effective dielectric constant $(\varepsilon=1.8)$ is considered for the computation in order to mimic the influence of the silicon nitride substrate on the LSPRs $(\varepsilon=4.0)$.

EELS Measurements. STEM-EELS maps were acquired using a FEI Titan Themis 80-300 equipped with a Wien-type monochromator and a Gatan GIF Quantum ERS spectrometer. A $300 \mathrm{keV}$ incident electron beam was used for all experiments, monochromated to give an energy spread of $\sim 110 \mathrm{meV}$ fwhm in the zero-loss peak of elastically scattered electrons, and with beam currents of $\sim 150$ to $230 \mathrm{pA}$. A $17 \mathrm{mrad}$ convergence semiangle of the probe and a 22 mrad collection semiangle on the spectrometer were used, with the probe having a mean diameter of $<1 \mathrm{~nm}$ for fwhm in incident intensity. Mapping was performed using the ultrafast spectrum imaging mode with typical dwell times of 0.25 to $0.5 \mathrm{~ms}$ per pixel and with the probe rastered in $\mathrm{X}, \mathrm{Y}$ step sizes of $0.5-0.6 \mathrm{~nm}$ for a total of $>105$ pixels per map. Each map was treated with the HQDark Correction plugin to reduce noise associated with dark current subtraction. Using this approach, the resultant plasmon maps have excellent spatial statistics when considering the inherently delocalized nature of this type of excitation.

EELS Processing. The EELS data cubes were processed using Gatan digital micrograph and custom Matlab scripts for the removal of the zero-loss radiation background and extraction of point spectra. The ZLP was first centered pixel by pixel using a Gaussian-Lorentzian approximation. The reflected tail model yielded a significantly lower signal-to-noise ratio and alignment accuracy, whereas fitting of a vacuum zeroloss offered no practical benefit while requiring a significantly longer computation time. Following zero-loss alignment, each data cube was spectrally cropped to the region of interest including ZLP ( -2 to $6 \mathrm{eV})$, and artifacts from cosmic rays were removed and the ZLP subtracted. Spectra in Figure 4 were integrated over a $30 \times 30$ pixel region of interest in the periphery of the discs.

\section{ASSOCIATED CONTENT}

\section{S Supporting Information}

The Supporting Information is available free of charge on the ACS Publications website at DOI: 10.1021/acsphotonics.6b00761.

Additional information (PDF)

\section{AUTHOR INFORMATION}

\section{Corresponding Author}

*E-mail: jeremy.butet@epfl.ch.

\section{ORCID ${ }^{\circ}$}

Jérémy Butet: 0000-0001-9598-9074

Valentin Flauraud: 0000-0002-1393-3198

Juergen Brugger: 0000-0002-7710-5930

Olivier J. F. Martin: 0000-0002-9574-3119

\section{Author Contributions}

G.D.B. developed and performed the numerical simulations with the standard formulation and edited the manuscript. J.Bu. developed and performed the numerical simulations with the antenna theory and wrote the manuscript. V.F. fabricated the samples, performed the STEM-EELS measurements, and edited the manuscript. D.T.L.A. performed the STEM-EELS measurements and edited the manuscript. J.Br. supervised the fabrication and edited the manuscript. O.J.F.M. supervised the project and edited the manuscript.

\section{Author Contributions}

${ }^{\#}$ G. D. Bernasconi and J. Butet contributed equally to this work. Notes

The authors declare no competing financial interest.

\section{ACKNOWLEDGMENTS}

The authors thank the staff of the Center of Micro/ Nanotechnology (CMI) of EPFL for the valuable discussions and support. This research was funded by the European Commission (FP7-ICT-2011-7, NANO-VISTA, under Grant Agreement No. 288263) and the Swiss National Science Foundation project 200020_153662.

\section{REFERENCES}

(1) Batson, P. E. Simultaneous STEM Imaging and Electron EnergyLoss Spectroscopy with Atomic-Column Sensitivity. Nature 1993, 366, $727-728$.

(2) Egerton, R. F. Electron Energy-Loss Spectroscopy in the TEM. Rep. Prog. Phys. 2009, 72, 016502.

(3) Browning, N. D.; Wallis, D. J.; Nellist, P. D.; Pennycook, S. J. EELS in the STEM: Determination of Materials Properties on the Atomic Scale. Micron 1997, 28, 333-348.

(4) Suenaga, K.; Tencé, M.; Mory, C.; Colliex, C.; Kato, H.; Okazaki, T.; Shinohara, H.; Hirahara, K.; Bandow, S.; Iijima, S. ElementSelective Single Atom Imaging. Science 2001, 290, 2280-2282.

(5) Varela, M.; Findlay, S. D.; Lupini, A. R.; Christen, H. M.; Borisevich, A. Y.; Dellby, N.; Krivanek, O. L.; Nellist, P. D.; Oxley, M. P.; Allen, L. J.; Pennycook, S. J. Spectroscopic Imaging of Single Atoms within a Bulk Solid. Phys. Rev. Lett. 2004, 92, 095502.

(6) Kimoto, K.; Asaka, T.; Nagai, T.; Saito, M.; Matsui, Y.; Ishizuka, K. Element-Selective Imaging of Atomic Columns in a Crystal using STEM and EELS. Nature 2007, 450, 702-704.

(7) Suenaga, K.; Sato, Y.; Liu, Z.; Kataura, H.; Okazaki, T.; Kimoto, K.; Sawada, H.; Sasaki, T.; Omoto, K.; Tomita, T.; Kaneyama, T.; Kondo, Y. Visualizing and Identifying Single Atoms using Electron Energy-Loss Spectroscopy with Low Accelerating Voltage. Nat. Chem. 2009, 1, 415-418.

(8) Krivanek, O. L.; Lovejoy, T. C.; Dellby, N.; Aoki, T.; Carpenter, R. W.; Rez, P.; Soignard, E.; Zhu, J.; Batson, P. E.; Lagos, M. J.; Egerton, R. F.; Crozier, P. A. Vibrational Spectroscopy in the Electron Microscope. Nature 2014, 514, 209-212.

(9) Crewe, A. V.; Isaacson, M.; Johnson, D. Electron Energy Loss Spectra of the Nucleic Acid Bases. Nature 1971, 231, 262-263.

(10) Taverna, D.; Kociak, M.; Stéphan, O.; Fabre, A.; Finot, E.; Décamps, B.; Colliex, C. Probing Physical Properties of Confined Fluids within Individual Nanobubbles. Phys. Rev. Lett. 2008, 100, 035301.

(11) Tan, H.; Turner, S.; Yücelen, E.; Verbeeck, J.; Tendeloo, G. V. 2D Atomic Mapping of Oxidation States in Transition Metal Oxides by Scanning Transmission Electron Microscopy and Electron EnergyLoss Spectroscopy. Phys. Rev. Lett. 2011, 107, 107602.

(12) Ramasse, Q. M.; Seabourne, C. R.; Kepaptsoglou, D.-M.; Zan, R; Bangert, U.; Scott, A. J. Probing the Bonding and Electronic Structure of Single Atom Dopants in Graphene with Electron Energy Loss Spectroscopy. Nano Lett. 2013, 13, 4989-4995.

(13) Senga, R.; Suenaga, K. Single-Atom Electron Energy Loss Spectroscopy of Light Elements. Nat. Commun. 2015, 6, 7943.

(14) García de Abajo, F. J. Optical Excitations in Electron Microscopy. Rev. Mod. Phys. 2010, 82, 209-275.

(15) Kociak, M.; Stéphan, O. Mapping Plasmons at the Nanometer Scale in an Electron Microscope. Chem. Soc. Rev. 2014, 43, 38653883. 
(16) Nelayah, J.; Kociak, M.; Stéphan, O.; García de Abajo, F. J.; Tencé, M.; Henrard, L.; Taverna, D.; Pastoriza-Santos, I.; Liz-Marzán, L. M.; Colliex, C. Mapping Surface Plasmons on a Single Metallic Nanoparticle. Nat. Phys. 2007, 3, 348-353.

(17) Raza, S.; Kadkhodazadeh, S.; Christensen, T.; Di Vece, M.; Wubs, M.; Mortensen, N. A.; Stenger, N. Multipole Plasmons and their Disappearance in Few-Nanometer Silver Nanoparticles. Nat. Commun. 2015, 6, 8788.

(18) Collins, S. M.; Midgley, P. A. Surface Plasmon Ecitations in Metal Spheres: Direct Comparison of Light Scattering and Electron Energy-Loss Spectroscopy by Modal Decomposition. Phys. Rev. B: Condens. Matter Mater. Phys. 2013, 87, 235432.

(19) Martin, J.; Kociak, M.; Mahfoud, Z.; Proust, J.; Gérard, D.; Plain, J. High-Resolution Imaging and Spectroscopy of Multipolar Plasmonic Resonances in Aluminum Nanoantennas. Nano Lett. 2014, 14, 55175523.

(20) Guiton, B. S.; Iberi, V.; Li, S.; Leonard, D. N.; Parish, C. M.; Kotula, P. G.; Varela, M.; Schatz, G. C.; Pennycook, S. J.; Camden, J. P. Correlated Optical Measurements and Plasmon Mapping of Silver Nanorods. Nano Lett. 2011, 11, 3482-3488.

(21) Rossouw, D.; Botton, G. A. Plasmonic Response of Bent Silver Nanowires for Nanophotonic Subwavelength Waveguiding. Phys. Rev. Lett. 2013, 110, 066801.

(22) Rossouw, D.; Couillard, M.; Vickery, J.; Kumacheva, E.; Botton, G. A. Multipolar Plasmonic Resonances in Silver Nanowire Antennas Imaged with a Subnanometer Electron Probe. Nano Lett. 2011, 11, 1499-1504.

(23) Schmidt, F. P.; Ditlbacher, H.; Hofer, F.; Krenn, J. R.; Hohenester, U. Morphing a Plasmonic Nanodisk into a Nanotriangle. Nano Lett. 2014, 14, 4810-4815.

(24) Schmidt, F. P.; Ditlbacher, H.; Hohenester, U.; Hohenau, A.; Hofer, F.; Krenn, J. R. Dark Plasmonic Breathing Modes in Silver Nanodisks. Nano Lett. 2012, 12, 5780-5783.

(25) Nicoletti, O.; de la Peña, F.; Leary, R. K.; Holland, D. J.; Ducati, C.; Midgley, P. A. Three-Dimensional Imaging of Localized Surface Plasmon Resonances of Metal Nanoparticles. Nature 2013, 502, 8084.

(26) Li, G.; Cherqui, C.; Bigelow, N. W.; Duscher, G.; Straney, P. J.; Millstone, J. E.; Masiello, D. J.; Camden, J. P. Spatially Mapping Energy Transfer from Single Plasmonic Particles to Semiconductor Substrates via STEM/EELS. Nano Lett. 2015, 15, 3465-347.

(27) Bellido, E. P.; Manjavacas, A.; Zhang, Y.; Cao, Y.; Nordlander, P.; Botton, G. A. Electron Energy-Loss Spectroscopy of Multipolar Edge and Cavity Modes in Silver Nanosquares. ACS Photonics 2016, 3, $428-433$.

(28) Myroshnychenko, V.; Nelayah, J.; Adamo, G.; Geuquet, N.; Rodriguez-Fernandez, J.; Pastoriza-Santos, I.; MacDonald, K. F.; Henrard, L.; Liz-Marzán, L. M.; Zheludev, N. I.; Kociak, M.; García de Abajo, F. J. Plasmon Spectroscopy and Imaging of Individual Gold Nanodecahedra: A Combined Optical Microscopy, Cathodoluminescence, and Electron Energy-Loss Spectroscopy Study. Nano Lett. 2012, 12, 4172-4180.

(29) Bosman, M.; Ye, E.; Tan, S. F.; Nijhuis, C. A.; Yang, J. K. W.; Marty, R.; Mlayah, A.; Arbouet, A.; Girard, C.; Han, M.-Y. Surface Plasmon Damping Quantified with an Electron Nanoprobe. Sci. Rep. 2013, 3, 1312.

(30) Collins, S. M.; Ringe, E.; Duchamp, M.; Saghi, Z.; DuninBorkowski, R. E.; Midgley, P. A. Eigenmode Tomography of Surface Charge Oscillations of Plasmonic Nanoparticles by Electron Energy Loss Spectroscopy. ACS Photonics 2015, 2, 1628-1635.

(31) von Cube, F.; Niegemann, J.; Irsen, S.; Bell, D. C.; Linden, S. Angular-Resolved Electron Energy Loss Spectroscopy on a Split-Ring Resonator. Phys. Rev. B: Condens. Matter Mater. Phys. 2014, 89, 115434.

(32) Koh, A. L.; Fernandez-Dominguez, A. I.; McComb, D. W.; Maier, S. A.; Yang, J. K. W. High-Resolution Mapping of ElectronBeam-Excited Plasmon Modes in Lithographically Defined Gold Nanostructures. Nano Lett. 2011, 11, 1323-1330.
(33) Kadkhodazadeh, S.; Rosenkrantz de Lasson, J.; Beleggia, M.; Kneipp, H.; Birkedal Wagner, J.; Kneipp, K. Scaling of the Surface Plasmon Resonance in Gold and Silver Dimers Probed by EELS. J. Phys. Chem. C 2015, 118, 5478-5485.

(34) Zhang, M.; Large, N.; Koh, A. L.; Cao, Y.; Manjavacas, A.; Sinclair, R.; Nordlander, P.; Wang, S. X. High-Density 2D Homo- and Hetero- Plasmonic Dimers with Universal Sub-10-nm Gaps. ACS Nano 2015, 9, 9331-9339.

(35) Scholl, J. A.; Garcia-Etxarri, A.; Aguirregabiria, G.; Esteban, R.; Narayan, T. C.; Koh, A. L.; Aizpurua, J.; Dionne, J. A. Evolution of Plasmonic Metamolecule Modes in the Quantum Tunneling Regime. ACS Nano 2016, 10, 1346-1354.

(36) Barrow, S. J.; Rossouw, D.; Funston, A. M.; Botton, G. A.; Mulvaney, P. Mapping Bright and Dark Modes in Gold Nanoparticle Chains using Electron Energy Loss Spectroscopy. Nano Lett. 2014, 14, 3799-3808.

(37) Coenen, T.; Schoen, D. T.; Mann, S. A.; Rodriguez, S. R. K.; Brenny, B. J. M.; Polman, A.; Brongersma, M. L. Nanoscale Spatial Coherent Control over the Modal Excitation of a Coupled Plasmonic Resonator System. Nano Lett. 2015, 15, 7666-7670.

(38) Gomez-Medina, R.; Yamamoto, N.; Nakano, M.; García de Abajo, F. J. Mapping Plasmons in Nanoantennas via Catholuminescence. New J. Phys. 2008, 10, 105009.

(39) Barnard, E. S.; Coenen, T.; Vesseur, E. J. R.; Polman, A.; Brongersma, M. L. Imaging the Hidden Modes of Ultrathin Plasmonic Strip Antennas by Cathodoluminescence. Nano Lett. 2011, 11, 42654269.

(40) Losquin, A.; Zagonel, L. F.; Myroshnychenko, V.; RodriguezGonzalez, B.; Tencé, M.; Scarabelli, L.; Förstner, J.; Liz-Marzán, L. M.; García de Abajo, F. J.; Stéphan, O.; Kociak, M. Unveiling Nanometer Scale Extinction and Scattering Phenomena through Combined Electron Energy Loss Spectroscopy and Cathodoluminescence Measurements. Nano Lett. 2015, 15, 1229-1237.

(41) Atre, A. C.; Brenny, B. J. M.; Coenen, T.; Garcia-Etxarri, A.; Polman, A.; Dionne, J. A. Nanoscale Optical Tomography with Cathodoluminescence Spectroscopy. Nat. Nanotechnol. 2015, 10, 429-436.

(42) Flauraud, V.; Mastrangeli, M.; Bernasconi, G. D.; Butet, J.; Alexander, D. T. L.; Shahrabi, E.; Martin, O. J. F.; Brugger, J. Nanoscale Topographical Control of Capillary Assembly of Nanoparticles. Nat. Nanotechnol. Accepted for publication. DOI: 10.1038/ nnano.2016.179.

(43) Sönnichsen, C.; Franzl, T.; Wilk, T.; von Plessen, G.; Feldmann, J.; Wilson, O.; Mulvaney, P. Drastic Reduction of Plasmon Damping in Gold Nanorods. Phys. Rev. Lett. 2002, 88, 077402.

(44) Geuquet, N.; Henrard, L. EELS and Optical Response of a Noble Metal Nanoparticle in the Frame of a Discrete Dipole Approximation. Ultramicroscopy 2010, 110, 1075.

(45) Bigelow, N. W.; Vaschillo, A.; Iberi, V.; Camden, J. P.; Masiello, D. J. Characterization of the Electron- and Photon-Driven Plasmonic Excitations of Metal Nanorods. ACS Nano 2012, 6, 7497-7504.

(46) Bigelow, N. W.; Vaschillo, A.; Camden, J. P.; Masiello, D. J. Signatures of Fano Interferences in the Electron Energy Loss Spectroscopy and Cathodoluminescence of Symmetry-Broken Nanorod Dimers. ACS Nano 2013, 7, 4511-4519.

(47) Arbouet, A.; Mlayah, A.; Girard, C.; Colas des Francs, G. Electron Energy Losses and Cathodoluminescence from Complex Plasmonic Nanostructures: Spectra, Maps and Radiation Patterns from a Generalized Field Propagator. New J. Phys. 2014, 16, 113012.

(48) Cao, Y.; Manjavacas, A.; Large, N.; Nordlander, P. Electron Energy-Loss Spectroscopy Calculation in Finite-Difference TimeDomain Package. ACS Photonics 2015, 2, 369-375.

(49) Matyssek, C.; Niegemann, J.; Hergert, W.; Busch, K. Computing Electron Energy Loss Spectra with the Discontinuous Galerkin TimeDomain Method. Phot. Nanostruc. Fundam. Appl. 2011, 9, 367-373.

(50) García de Abajo, F. J.; Aizpurua, J. Numerical Simulation of Electron Energy Loss Near Inhomogeneous Dielectrics. Phys. Rev. B: Condens. Matter Mater. Phys. 1997, 56, 15873-15884. 
(51) García de Abajo, F. J.; Howie, A. Relativistic Electon Energy Loss and Electron-Induced Photon Emission in Inhomogeneous Dielectrics. Phys. Rev. Lett. 1998, 80, 5180-5183.

(52) García de Abajo, F. J.; Howie, A. Retarded Field Calculation of Electron Energy Loss in Inhomogeneous Dielectrics. Phys. Rev. B: Condens. Matter Mater. Phys. 2002, 65, 115418.

(53) Hohenester, U.; Trügler, A. MNPBEM - A Matlab Toolbox for the Simulation of Plasmonic Nanoparticles. Comput. Phys. Commun. 2012, 183, 370.

(54) García de Abajo, F. J.; Kociak, M. Probing the Photonic Local Density of States with Electron Energy Loss Spectroscopy. Phys. Rev. Lett. 2008, 100, 106804.

(55) Hohenester, U.; Ditlbacher, H.; Krenn, J. Electron Energy Loss Spectroscopy of Plasmonic Nanoparticles. Phys. Rev. Lett. 2009, 103, 106801.

(56) Boudarham, G.; Kociak, M. Modal Decompositions of the Local Electromagnetic Density of States and Spatially Resolved Electron Energy Loss Probability in Terms of Geometric Modes. Phys. Rev. B: Condens. Matter Mater. Phys. 2012, 85, 245447.

(57) Losquin, A.; Kociak, M. Link between Cathodoluminescence and Electron Energy Loss Spectroscopy and the Radiative and Full Electromagnetic Local Density of States. ACS Photonics 2015, 2, 1619-1627.

(58) Hörl, A.; Trügler, A.; Hohenester, U. Tomography of Particle Plasmon Fields from Electron Eergy Loss Spectroscopy. Phys. Rev. Lett. 2013, 111, 086801.

(59) Hörl, A.; Trügler, A.; Hohenester, U. Full Three-Dimensonal Reconstruction of the Dyadic Green Tensor from Electron Energy Loss Spectroscopy of Plasmonic Nanoparticles. ACS Photonics 2015, 2, 1429-1435.

(60) Zhu, D.; Bosman, M.; Yang, J. K. W. A Circuit Model for Plasmonic resonators. Opt. Express 2014, 22, 9809-9819.

(61) Duan, H.; Fernández-Domínguez, A. I.; Bosman, M.; Maier, S. A.; Yang, J. K. L. Nanoplasmonics: Classical down to the Nanometer Scale. Nano Lett. 2012, 12, 1683-1689.

(62) García de Abajo, F. J.; Rivacoba, A.; Zabala, N.; Yamamoto, N. Boundary effects in Cherenkov Radiation. Phys. Rev. B: Condens. Matter Mater. Phys. 2004, 69, 155420.

(63) Mühlschlegel, P.; Eisler, H.-J.; Martin, O. J. F.; Hecht, B.; Pohl,

D. W. Resonant Optical Antennas. Science 2005, 308, 1607-1608.

(64) Kern, A. M.; Martin, O. J. F. Surface Integral Formulation for 3D Simulation of Plasmonic and High Permittivity Nanostructures. J. Opt. Soc. Am. A 2009, 26, 732-740.

(65) Jackson, J. D. Classical Electrodynamics, 2nd ed.; Wiley: New York, 1975.

(66) Kern, A. M.; Martin, O. J. F. Excitation and Reemission of Molecules Near Realistic Plasmonic Nanostructures. Nano Lett. 2011, 11, 482-487.

(67) Ritchie, R. H. Plasma Losses by Fast Electrons in Thin Film. Phys. Rev. 1957, 106, 874-881.

(68) Gallinet, B.; Butet, J.; Martin, O. J. F. Numerical Methods for Nanophotonics: Standard Problems and Future Challenges. Laser. Phot. Rev. 2015, 9, 577-603.

(69) Brongersma, M. L.; Halas, N. J.; Nordlander, P. PlasmonInduced Hot Carrier Science and Technology. Nat. Nanotechnol. 2015, $10,25-34$.

(70) Knight, M. W.; Sobhani, H.; Nordlander, P.; Halas, N. J. Photodetection with Active Optical Antennas. Science 2011, 332, 702704.

(71) Kern, A. M.; Martin, O. J. F. Pitfalls in the Determination of Optical Cross Sections from Surface Integral Equation Simulations. IEEE Trans. Antennas Propag. 2010, 58, 2158-2161.

(72) Johnson, P. B.; Christy, R. W. Optical Constants of the Noble Metals. Phys. Rev. B 1972, 6, 4370-4379.

(73) Batson, P. E. Surface Plasmon Coupling in Clusters of Small Spheres. Phys. Rev. Lett. 1982, 49, 936.

(74) Bernasconi, G. D.; Butet, J.; Martin, O. J. F. Mode Analysis of Second-Harmonic Generation in Plasmonic Nanostructures. J. Opt. Soc. Am. B 2016, 33, 768-779.
(75) Luk'yanchuck, B.; Zheludev, N. I.; Maier, S. A.; Halas, N. J.; Nordlander, P.; Giessen, H.; Chong, C. T. The Fano Resonance in Plasmonic Nanostructures and Metamaterials. Nat. Mater. 2010, 9, $707-715$. 